Revue

Revue de l'histoire des religions

de Ihistoire

des religions

$3 \mid 2006$

Varia

\title{
Haim Goren, "Zieht aus und erforscht das Land »: Die deutsche Palästina-Forschung im 19. Jahrhundert
}

Wallstein, Göttingen, 2003

Dominique Trimbur

\section{(2) OpenEdition \\ Journals}

Édition électronique

URL : http://journals.openedition.org/rhr/5177

DOI : $10.4000 /$ rhr. 5177

ISSN : 2105-2573

Éditeur

Armand Colin

Édition imprimée

Date de publication : 1 septembre 2006

Pagination : 352-354

ISBN : 2200-92105-5

ISSN : 0035-1423

Référence électronique

Dominique Trimbur, " Haim Goren, "Zieht aus und erforscht das Land »: Die deutsche Palästina-

Forschung im 19. Jahrhundert », Revue de l'histoire des religions [En ligne], 3 | 2006, mis en ligne le 22

janvier 2010, consulté le 22 septembre 2020. URL : http://journals.openedition.org/rhr/5177 ; DOI :

https://doi.org/10.4000/rhr.5177

Ce document a été généré automatiquement le 22 septembre 2020.

Tous droits réservés 


\title{
Haim Goren, « Zieht aus und erforscht das Land »: Die deutsche Palästina- Forschung im 19. Jahrhundert
}

\author{
Wallstein, Göttingen, 2003
}

Dominique Trimbur

\section{RÉFÉRENCE}

Haim Goren, «Zieht aus und erforscht das Land »: Die deutsche Palästina-Forschung im 19.

Jahrhundert, Wallstein, Göttingen, 2003, 432 p.

Depuis près de trois décennies, l'historiographie israélienne se dégage du «sionistocentrisme » qui, par souci d'affirmation identitaire et nationale, avait majoritairement passé sous silence la contribution européenne au développement de la «Palestine » et de la «Terre sainte» (concepts également tus pour leur préférer celui de «Eretz Israël »). Mettant fin à une conception exclusive selon laquelle les Juifs et les sionistes avaient constitué les seuls éléments de développement de la région, il est désormais acquis que d'autres facteurs avaient contribué positivement à cette évolution. L'ouvrage de Haim Goren, d'abord paru en hébreu en 1999 et sorti en allemand en 2003, participe de cette " redécouverte ».

2 C'est justement à la description d'une des facettes de la «redécouverte» ou de l'« invention » de la Terre sainte que s'attelle l'auteur: il retrace, dans une étude très solidement documentée, l'élan allemand en direction de la Palestine. Directement issu du mouvement romantique, comme ses équivalents français ou anglais, il consiste en une accumulation d'efforts à la fois scientifiques et religieux visant à une exploration approfondie du terrain de l'Évangile.

3 À travers une série de portraits détaillés, par un inventaire précis (parfois un peu répétitif), l'auteur nous offre un tableau complet, voire exhaustif, de l'exploration allemande de la Terre sainte. De courtes biographies, regroupées par périodes, par 
origines géographiques (lieux d'activité des scientifiques - universités ou académies des sciences), par confessions (protestants et catholiques, ces derniers étant moins audacieux que les premiers en matière d'avancées scientifiques, à l'exception peut-être de Johann Nepomuk Sepp), retracent une constellation aux multiples composantes : les chercheurs, souvent concentrés en des villes phares (Göttin-gen, Berlin, Munich, Leipzig, Halle), et, placés dans des relations de maîtres à disciples, constituant de véritables générations, entretiennent des relations très étroites, constituant ainsi des réseaux particulièrement serrés et efficaces. Se retrouvent ici des chercheurs, des plus connus (Carl Ritter, Titus Tobler, Conrad Schick...) aux plus modestes, aux cursus très proches, faits d'un passage obligé par certaines universités allemandes et d'un séjour non moins obligatoire à Paris pour suivre, au Collège de France, les cours de Sacy, par exemple; des descendants des érudits des siècles précédents, cumulant les connaissances et les domaines d'activités (géographie, géologie, sciences naturelles, architecture, archéologie, ethnologie, histoire, épigraphie, science biblique, dessin, mystique et poésie...) ; des savants qui diffusent leur savoir par la rédaction d'articles ou de sommes, qui collectent des données de toutes sortes, créent des revues comme des sociétés savantes...

4 La démonstration - magistrale - de Haim Goren retrace la naissance et l'évolution d'une aventure scientifique. L'auteur souligne ainsi l'élaboration progressive d'un objet d'étude, car la "redécouverte» de la Terre sainte du point de vue politico-religieux s'accompagne de sa découverte scientifique. La Palestine met du temps à représenter une région sur laquelle vont se focaliser les scientifiques, qui jusque-là ne font que la traverser au cours de périples n'envisageant la région que comme un sous-ensemble d'un Orient plus large (Carsten Niebuhr ou Johann Ludwig Buckhardt). Par ailleurs, à une époque où les transports sont loin du confort actuel, certains chercheurs mettent du temps à entreprendre réellement le voyage, plusieurs d'entre eux n'hésitant pas à s'attaquer à une description de la région à partir de leur table de travail.

5 Cette recherche est ensuite incomparable par ses éléments constitutifs : mélange de foi et de science, elle se trouve à la croisée de multiples enjeux. Il en va d'un travail religieux, puisque la plupart des scientifiques se spécialisent sur la Terre sainte pour appuyer par leur science les textes bibliques; le plus souvent également missionnaires, leur recherche doit apporter les preuves de la réalité des récits évangéliques (mais elle peut aussi parfois faire encourir le risque de mettre à jour certaines contradictions, à l'instar des travaux de cartographie de Heinrich Kiepert). Ce travail est également une œuvre largement politique, voire militaire: la «redécouverte» de la Terre sainte s'accompagne d'une rivalité croissante entre les puissances du moment. Le retour en ces contrées est, aux yeux de beaucoup, une possibilité de procéder à une nouvelle croisade, pacifique cette fois; et de la même manière que les missionnaires et autres religieux deviennent des instruments pratiques pour des ambitions politiques très pragmatiques, les scientifiques doivent œuvrer pour le bien de leurs nations d'origine. C'est évidemment le cas des personnes décrites par Haim Goren, avec des princes et rois allemands, puis un empereur d'Allemagne, qui les utilisent de façon affichée. En ce sens, la découverte et l'exploration scientifique est une appropriation aussi bien religieuse que politique ; et une œuvre de mécénat permet de pratiquer une politique de prestige défiant les puissances rivales, action culturelle à l'étranger avant l'heure. Il n'est d'ailleurs pas surprenant que les premiers consuls de Prusse à Jérusalem soient des orientalistes, maîtrisant parfaitement les langues locales et aux connaissances scientifiques poussées (Ernst Gustav Schultz ou Georg Rosen, entre autres). De façon 
pragmatique, ces recherches sont autant de premiers repérages pour une œuvre de développement des institutions religieuses allemandes, catholiques ou protestantes ; si ce n'est, plus franchement, de colonisation (Philipp Wolff), dans le but d'installer là des Juifs ou des chrétiens. On a donc insertion de l'entreprise scienti-fico-religieuse dans une perspective proto-coloniale : l'association de certains scientifiques avec les Templer, par exemple, vise clairement à faire pièce aux efforts russes, notamment.

C'est aussi ce qui progressivement mène à la prise en compte de la Palestine réelle : le dépassement de la religiosité s'accompagne ainsi du développement des sciences appliquées (des travaux géologiques devant offrir une place plus importante à l'économie allemande dans la région). Il n'est pas étonnant de ce point de vue que l'on assiste progressivement à une rationalisation : de la même manière que se met en place une "pales-tinologie» (Palästinawissenschaft) en tant que telle, celle-ci se professionnalise peu à peu. Il en va de la nécessité d'échapper à l'amateurisme romantique qui avait caractérisé les premiers pas de cette science nouvelle pour lui donner une apparence plus sérieuse; il en va également de la nécessité de concentrer et d'harmoniser les efforts, au service de la religion, de la science et de la politique. Les chercheurs allemands de toutes origines, électrons plus ou moins dispersés et libres au départ, se trouvent ainsi insérés dans des institutions scientifiques établies dans la deuxième moitié du XIX siècle, et qui existent encore aujourd'hui : sur le modèle du Palestine Exploration Fund britannique (1865) sont créés le Deutscher Palästina Verein (1878), sa revue et son instrument scientifique, le Deutsch-evangelisches Institut für Altertumswissenchaften des Heiligen Landes (1902), placé sous la direction de Gustav Dalman ; tandis que du côté catholique est établie à Jérusalem l'antenne de la Görres Gesellschaft en 1909.

7 Si l'on peut regretter certaines ellipses ou approximations, notamment en ce qui concerne les domaines où l'auteur est moins à l'aise (en particulier le domaine français), si l'on peut aussi souligner un recul parfois insuffisant envers sa matière (avec une conclusion trop brève, ne mettant guère en exergue les apports de cet énorme travail), il faut saluer ce livre comme constituant une contribution désormais incontournable à notre connaissance de la Palestine du xIX siècle, aux relations scientifiques, religieuses et politiques (étroitement imbriquées entre elles). Il offre un nouvel éclairage, positif cette fois, sur une facette de l'orientalisme tant décrié par Edward Saïd. Son apport est d'autant plus grand qu'il fait regretter l'absence d'une telle étude concernant la "palestinologie» française : incarnée par certaines institutions célèbres, comme l'École biblique dont l'histoire a en grande partie été retracée, elle est bel et bien essentielle pour les scientifiques actuels comme pour les contemporains des événements relatés ici.

\section{AUTEUR}

\section{DOMINIQUE TRIMBUR}

Centre de Recherche français de Jérusalem. 